Bull. Austral. Math. Soc.

VOL. 37 (1988) [367-378]

\title{
MULTIOBJECTIVE FRACTIONAL DUALITY
}

\author{
RICHARD R. Egudo
}

The concept of efficiency (Pareto optimum) is used to formulate duality for multiobjective fractional programming problems. We consider programs where the components of the objective function have non-negative and convex numerators while the denominators are concave and positive. For this case the Mond-Weir extension of Bector dual analogy is given. We also give the Schaible type vector dual. The case where functions are $\rho$-convex (weakly or strongly convex) is also considered.

\section{INTRODUCTION AND PRELIMINARIES}

Consider the following multiobjective fractional programming problem:

(FP):

$$
\text { Minimise }\left(\frac{f_{1}(x)}{g_{1}(x)}, \frac{f_{2}(x)}{g_{2}(x)}, \ldots, \frac{f_{p}(x)}{g_{p}(x)}\right)
$$

subject to

$$
h(x) \leqq 0 ;
$$

where $f_{i}: \mathbf{R}^{n} \rightarrow \mathbf{R}, g_{i}: \mathbf{P}^{n} \rightarrow \mathbf{R}$ for $i=1,2, \ldots, p$ and $h: \mathbf{R}^{n} \rightarrow \mathbf{R}^{m}$ are differentiable functions and minimisation entails obtaining efficient solutions.

Definition 1. A feasible solution $x^{0}$ for (FP) is an efficient solution for (FP) if there exists no other feasible solution $x$ for (FP) such that for some $i \in P=\{1,2, \ldots, p\}$, $\frac{f_{i}(x)}{g_{i}(x)}<\frac{f_{i}\left(x^{0}\right)}{g_{i}\left(x^{0}\right)}$ and $\frac{f_{j}(x)}{g_{j}(x)} \leqq \frac{f_{j}\left(x^{0}\right)}{g_{j}\left(x^{0}\right)}$ for all $j \in P$.

In the case of maximisation problems the signs in the above two inequalities are reversed.

In [11], Singh gave a duality result for a class of multiobjective fractional programs (FP), where he assumed that among the denoninators $g_{1}, g_{2}, \ldots, g_{p}$, there is one which when multiplied by the other components (that is, $f_{i} / g_{i}, i=1,2, \ldots, p$ ) gives convex functions. Also Weir [15], considered a special class of (FP) whereby all the denominators are equal to $g(x)$. Recently Weir [14] used proper efficienty [5], to obtain a dual to (FP) when $f_{i}(x) \geqq 0$ and convex, and $g_{i}(x)>0$ and concave for each $i=1,2, \ldots, p$. Weir's dual [14], is simpler than Singh [11] in that it does away

Received 24 July, 198i

C'opyright Clearance Centre, Inc. Serial-fee code: 0004-9729/88 \$A2.00+0.00. 
with the condition of identifying a denominator which produces convex functions when multiplied with the objective function components. It also generalises Singh [11] in the sense that a program which satisfies Weir conditions [14] may fail to satisfy conditions required by Singh [11]. Weir's dual [14] is an analogue of the Mond-Weir [8] extension of the Bector dual [2] in the single objective non-linear programming case.

In this paper we use the concept of efficiency (Pareto optimum) rather than proper efficiency to formulate duality between (FP) and the following two multiobjective nonlinear programming problems:

\section{Weir type dual.}

(FD1):

$$
\underset{u, \tau, y}{\operatorname{Maximise}}\left(\frac{f_{1}(u)}{g_{1}(u)}, \frac{f_{2}(u)}{g_{2}(u)}, \ldots \frac{f_{p}(u)}{g_{p}(u)}\right)
$$

subject to

$$
\tau_{i} \geqq 0, \quad i=1,2, \ldots, p, \quad \sum_{i=1}^{p} \tau_{i}=1
$$

and

Schaible type dual.

(FD2):

$$
\underset{u, r, y}{\operatorname{Maximise}}\left(\lambda_{1}, \lambda_{2}, \ldots, \lambda_{p}\right)
$$

subject to

$$
\begin{gathered}
\sum_{i=1}^{p} \tau_{i}\left(\nabla f_{i}(u)-\lambda_{i} g_{i}(u)\right)+\nabla y^{i} h(u)=0 \\
\frac{f_{i}(u)}{g_{i}(u)}=\lambda_{i}, \quad i=1,2, \ldots, p
\end{gathered}
$$

and (3) to (5).

The program (FD1) is slightly more general than Weir's dual [14] in that we no longer require all the objective component multipliers $\tau_{i}$ 's to be strictly positive but they should be non-negative with at least one of them strictly positive. This relaxation is possible because instead of using proper efficiency we use efficiency to formulate duality relations between (FP) and (FD1). The program (FD2) is our multiobjective analogue of the Mond-Weir [8] extension of the Schaible $[9,10]$ dual in a single objective optimisation.

The following result will be required in the proofs of strong duality results. 
LEMMA 1. $x^{0}$ is an efficient solution for (FP) if and only if $x^{0}$ solves

$\left(\operatorname{FP}_{k}\left(\varepsilon^{0}\right)\right): \quad \quad$ Minimise $\frac{f_{k}(x)}{g_{k}(x)}$

subject to

$$
\begin{gathered}
\frac{f_{j}(x)}{g_{j}(x)} \leqq \varepsilon_{j}^{0} \text { for all } j \neq k \\
h(x) \leqq 0
\end{gathered}
$$

where $\varepsilon_{j}^{0}=f_{j}\left(x^{0}\right) / g_{j}\left(x^{0}\right) ;$ for each $k=1,2, \ldots, p$.

The proof of Lemma 1 is the same as the proof of Theorem 4.1 of Chankong and Haimes [3].

If $g_{j}(x)>0$, for each $j=1,2, \ldots, p$, then $\mathrm{FP}_{k}\left(\varepsilon^{0}\right)$ can be rewritten as

$\left(\operatorname{FP}_{k}^{\prime}\left(\varepsilon^{0}\right)\right): \quad \quad$ Minimise $\frac{f_{k}(x)}{g_{k}(x)}$

subject to

$$
\begin{gathered}
f_{j}(x)-\varepsilon_{j}^{0} g_{j}(x) \leqq 0 \text { for all } j \neq k \\
h(x) \leqq 0
\end{gathered}
$$

Lemma 2. Assume that $g_{j}(x)>0$ for each $j=1,2, \ldots, p$ in (FP). Then $x^{0}$ is an efficient solution of $(F P)$ if and only if $x^{0}$ solves $F P_{k}^{\prime}\left(\varepsilon^{0}\right)$ for each $k=1,2, \ldots, p$.

Proof: Let $x^{0}$ be an efficient solution for (FP) and suppose that $x^{0}$ does not solve $\mathrm{FP}_{k}^{\prime}\left(\varepsilon^{0}\right)$ for some $k \in P=\{1,2, \ldots, p\}$. Then there exists a feasible $x$ for (FP) such that

$$
\frac{f_{k}(x)}{g_{k}(x)}<\frac{f_{k}\left(x^{0}\right)}{g_{k}\left(x^{0}\right)}
$$

and

$$
f_{j}(x)-\varepsilon_{j}^{0} g_{j}(x) \leqq 0 \text { for all } j \neq k
$$

Since $g_{j}(x)>0$ for all $j=1,2, \ldots, p$, we rewrite $(9)$ as

$$
\frac{f_{j}(x)}{g_{j}(x)} \leqq \varepsilon_{j}^{0}=\frac{f_{j}\left(x^{0}\right)}{g_{j}\left(x^{0}\right)} \text { for all } j \neq k .
$$


Now (8) and (10) contradict the efficiency of $x^{0}$ for (FP).

To show the converse, assume that $x^{0}$ solves $\mathrm{FP}_{k}^{\prime}\left(\varepsilon^{0}\right)$ for all $k \in P$. Then there is no feasible $x$ in (FP) such that for some $k \in P$

$$
\frac{f_{k}(x)}{g_{k}(x)}<\frac{f_{k}\left(x^{0}\right)}{g_{k}\left(x^{0}\right)}
$$

and

$$
\frac{f_{j}(x)}{g_{j}(x)} \leqq \frac{f_{j}\left(x^{0}\right)}{g_{j}\left(x^{0}\right)} \text { for all } j \in P
$$

which is the definition of efficieny of $x^{0}$ for (FP).

\section{WEIR TYPE DUALITY}

In this section we prove weak and strong duality results between (FP) and (FD1).

Theorem 1 (Weak DUality). Assume that for all feasible $x$ in (FP) and all $(u, \tau, y)$ in $(F D 1), f_{i}$ is non-negative and convex, $g_{i}$ is positive and concave for each $i=$ $1,2, \ldots, p$; and that $y^{t} h$ is quasiconvex at $u$. If also either of the following hypotheses holds:

(a) $\tau_{i}>0$ for all $i \in P=\{1,2, \ldots, p\}$,

(b) $\sum_{i=1}^{p} \tau_{i} \frac{f_{i}(\bullet)}{g_{i}(\bullet)}$ is strictly pseudoconvex at $u$;

then the following cannot hold:

$$
\frac{f_{j}(x)}{g_{j}(x)} \leqq \frac{f_{j}(u)}{g_{j}(u)} \text { for all } j \in P
$$

and

$$
\frac{f_{i}(x)}{g_{i}(x)}<\frac{f_{i}(u)}{g_{i}(u)} \text { for some } i \in P \text {. }
$$

Proof: Since $x$ is feasible for (FP) and $(u, \tau, y)$ is feasible for (FD1) $y^{t} h(x)-$ $y^{t} h(u) \leqq 0$; and since $y^{t} h$ is quasiconvex at $u$, this inequality implies

$$
(x-u)^{t} \nabla y^{t} h(u) \leqq 0 .
$$

Applying (13) to (2) we obtain

$$
(x-u)^{t} \sum_{i=1}^{p} \tau_{i} \nabla \frac{f_{i}(u)}{g_{i}(u)} \geqq 0 .
$$


Now suppose contrary to the result of the theorem, that (11) and (12) hold. From lyypothesis (a) it follows that

$$
\tau_{i} \frac{f_{i}(x)}{g_{i}(x)}<\tau_{i} \frac{f_{i}(u)}{g_{i}(u)} \text { for some } i \in P
$$

and

$$
\tau_{j} \frac{f_{j}(x)}{g_{j}(x)} \leqq \tau_{j} \frac{f_{j}(u)}{g_{j}(u)} \text { for all } j \in P
$$

Also from $f_{j}(x) \geqq 0$ and $g_{j}(x)>0$ for all $j \in P$ it follows that $\tau_{j} \frac{f_{j}(x)}{g_{j}(x)}, j=1,2, \ldots, p$ are pseudoconvex (see $[\boldsymbol{1}, \boldsymbol{6}]$ for example).

Hence (15) and (16) imply

$$
(x-u)^{\iota} \sum_{i=1}^{p} \tau_{i} \nabla \frac{f_{i}(u)}{g_{i}(u)}<0
$$

which contradicts (14).

Applying $\tau_{i} \geqq 0, i=1,2, \ldots, p$ (since $\tau$ is feasible in (FD1)) to (11) and (12) we obtain

$$
\sum_{i=1}^{p} \tau_{i} \frac{f_{i}(x)}{g_{i}(x)} \leqq \sum_{i=1}^{p} \tau_{i} \frac{f_{i}(u)}{g_{i}(u)}
$$

Hypothesis (b) and (17) now imply

$$
(x-u)^{t} \sum_{i=1}^{p} r_{i} \nabla \frac{f_{i}(u)}{g_{i}(u)}<0
$$

again contradicting (14).

Remark 1. Weir [14] proved a similar result but he considered the case where only hypothesis (a) holds.

COROLlary 1. Let the conditions of weak duality (Theorem 1) hold. Then if $\left(u^{0}, \tau^{0}, y^{0}\right)$ is a feasible solution for (FD1) such that $u^{0}$ is also feasible for (FP), then $u^{0}$ is efficient for $(F P)$ and $\left(u^{0}, \tau^{0}, y^{0}\right)$ is efficient for (FD1).

Proof: The proof is by contradiction. Suppose that $u^{0}$ is not efficient for (FP), then there exists a feasible $x$ in (FP) such that for some $i \in P=\{1,2, \ldots, p\}$

$$
\frac{f_{i}(x)}{g_{i}(x)}<\frac{f_{i}\left(u^{0}\right)}{g_{i}\left(u^{0}\right)}
$$


and

$$
\frac{f_{j}(x)}{g_{j}(x)} \leqq \frac{f_{j}\left(u^{0}\right)}{g_{j}\left(u^{0}\right)} \text { for all } j \in P .
$$

Now since $\left(u^{0}, \tau^{0}, y^{0}\right)$ is feasible for (FD1), these inequalities contradict the weak duality (Theorem 1) result. Therefore $u^{0}$ must be an efficient solution for (FP). Similarly assuming that $\left(u^{0}, \tau^{0}, y^{0}\right)$ is not efficient for (FD1) leads to a contradiction; hence $\left(u^{0}, \tau^{0}, y^{0}\right)$ is efficient for (FD1).

Theorem 2 (STRONG DUALITY). Let $x^{0}$ be an efficient solution for (FP) and assume that $x^{0}$ satisfies a constraint qualification $[1,6]$ for $F_{k} P\left(\varepsilon^{0}\right)$ for at least one $k=1,2, \ldots, p$. Then there exists $\tau^{0} \in \mathbb{R}^{p}$ and $y^{0} \in \mathbb{R}^{m}$ such that $\left(x^{0}, \tau^{0}, y^{0}\right)$ is a feasible solution for (FD1). If also weak duality (Theorem 1) holds between (FD1) and $(F P)$ then $\left(x^{0}, \tau^{0}, y^{0}\right)$ is an efficient solution for (FD1).

Proof: Since $x^{0}$ is efficient for (FP), from Lemma $1, x^{0}$ solves $\mathrm{FP}_{k}\left(\varepsilon^{0}\right)$ for each $k \in P=\{1,2, \ldots p\}$. By hypothesis there exists a $k \in P$ such that $x^{0}$ satisfies a constraint qualification for $\mathrm{FP}_{k}\left(\varepsilon^{0}\right)$. From the Kuln-Tucker necessary conditions $[\mathbf{1}$, 0] there exist $\tau_{i} \geqq 0$ for all $i \neq k$, and $y \geqq 0 \in \mathbf{R}^{m}$ such that

$$
\begin{aligned}
\nabla \frac{f_{k}\left(x^{0}\right)}{g_{k}\left(x^{0}\right)}+\sum_{i \neq k} \tau_{i} \nabla \frac{f_{i}\left(x^{0}\right)}{g_{i}\left(x^{0}\right)}+\nabla y^{t} h\left(x^{0}\right) & =0 \\
y^{t} h\left(x^{0}\right) & =0 .
\end{aligned}
$$

Dividing all terms in (18) and (19) by $1+\sum_{i \neq k} \tau_{i}$ and setting $\tau_{k}^{0}=\frac{1}{1+\sum_{i \neq k} \tau_{i}}>0$, $\tau_{j}^{0}=\frac{\tau_{j}}{1+\sum_{i \neq k} \tau_{i}} \geqq 0$, and $y^{0}=\frac{y}{1+\sum_{i \neq k} \tau_{i}} \geqq 0$, we conclude that $\left(x^{0}, \tau^{0}, y^{0}\right)$ is a feasible solution for (FD1). Since weak duality (Theorem 1) holds between (FP) and (FD1), efficiency of $\left(x^{0}, \tau^{0}, y^{0}\right)$ for (FD1) now follows from Corollary 1.

\section{SCHAIBLE TYPE DUALITY}

In this section we prove weak and strong duality results between (FP) and (FD2). As in the previous section we assume that the numerators of the objective function components are non-negative and the denominators are strictly positive. The results will be given for convex, $\rho$-convex and quasiconvex functions.

Definition 2. A function $f: \mathbf{R}^{n} \rightarrow \mathbf{R}$ is $\rho$-convex, $[12,13]$, if there exists a. real number $\rho$ such that for each $x, u \in \mathbf{R}^{n}$ and $0 \leqq \lambda \leqq 1$,

$$
f(\lambda x+(1-\lambda) u) \leqq \lambda f(x)+(1-\lambda) f(u)-\rho \lambda(1-\lambda)\|x-u\|^{2} .
$$


For a differentiable function $f: \mathbf{R}^{n} \rightarrow \boldsymbol{R}, f$ is $\rho$-convex [13] if and only if for each $x, u \in \mathbf{R}^{n}$

$$
f(x)-f(u) \geqq(x-u)^{t} \nabla f(u)+\rho\|x-u\|^{2} .
$$

When $\rho$ is positive $f$ is said to be strongly convex [13] and if $\rho$ is negative it is said to be weakly convex; and of course $f$ is convex if $\rho=0$.

Theorem 3 (Weak DUality). Assume that for each feasible $x$ for (FP) and each feasible $(u, \tau, y)$ for $(F D 2) ; f_{i}(x) \geqq 0$ and convex, $g_{i}(x)>0$ and concave for each $i=1,2, \ldots, p$; and that $y^{t} h$ is quasiconvex at $u$. If either of the following holds:

(a) $\tau_{i}>0$ for all $i \in P=\{1,2, \ldots, p\}$,

(b) $\sum_{i=1}^{p} \tau_{i}\left(f_{i}-\lambda_{i} g_{i}\right)$ is strictly convex at $u$;

then the following cannot hold:

$$
\frac{f_{j}(x)}{g_{j}(x)} \leqq \frac{f_{j}(u)}{g_{j}(u)} \text { for all } j \in P
$$

and

$$
\frac{f_{i}(x)}{g_{i}(x)}<\frac{f_{i}(u)}{g_{i}(u)} \text { for some } i \in P
$$

Proof: From the feasibility of $x$ and $(u, \tau, y)$ in (FP) and (FD2) respectively, we have $y^{t} h(x)-y^{t} h(u) \leqq 0$; and since $y^{t} h$ is quasiconvex at $u$, this inequality implies

$$
(x-u)^{\iota} \nabla y^{t} h(u) \leqq 0
$$

Now suppose contrary to the result of the theorem, that (20) and (21) hold for some feasible $x$ for (FP) and $(u, \tau, y)$ for (FD2). But $\frac{f_{j}(u)}{g_{j}(u)}=\lambda_{j}$ and $g_{j}(x)>0$ for each $j=1,2, \ldots, p$. So that $(20)$ and $(21)$ can be rewritten as

$$
f_{j}(x)-\lambda_{j} g_{j}(x) \leqq f_{j}(u)-\lambda_{j} g_{j}(u) \text { for all } j \in P
$$

and

$$
f_{i}(x)-\lambda_{i} g_{i}(x)<f_{i}(u)-\lambda_{i} g_{i}(u) \text { for some } i \in P
$$

respectively. If hypothesis (a) holds, that is, $\tau_{j}>0$ for each $j \in P$ then (23) and (24) imply

$$
\tau_{j}\left(f_{j}(x)-\lambda_{j} g_{j}(x)\right) \leqq \tau_{j}\left(f_{j}(u)-\lambda_{j} g_{j}(u)\right) \text { for all } j \in P
$$


and

$$
\tau_{i}\left(f_{i}(x)-\lambda_{i} g_{i}(x)\right)<\tau_{i}\left(f_{i}(u)-\lambda_{i} g_{i}(u)\right) \text { for some } i \in P
$$

respectively. From $\lambda_{j} \geqq 0, \tau_{j}>0$ and the convexity of $f_{j}$ and $-g_{j}$ for each $j \in P$ we know that $\tau_{j}\left(f_{j}(\bullet)-\lambda_{j} g_{J}(\bullet)\right)$ is convex for each $j \in P$. Therefore inequalities (25) and (26) imply

$$
(x-u)^{t} \sum_{i=1}^{p} \tau_{i} \nabla\left(f_{i}(u)-\lambda_{i} g_{i}(u)\right)<0
$$

Adding (22) to (27) we obtain

$$
(x-u)^{t}\left(\sum \tau_{i} \nabla\left(f_{i}(u)-\lambda_{i} g(u)\right)+\nabla y^{t} h(u)\right)<0,
$$

contradicting $(6)$.

If hypothesis (b) holds, then we apply $\tau_{j} \geqq 0, j=1,2, \ldots, p$ (since $\tau$ is feasible in (FD2)) to (23) and (24) to obtain

$$
\sum_{j=1}^{p} \tau_{j}\left(f_{j}(x)-\lambda_{j} g_{j}(x)\right) \leqq \sum_{j=1}^{p} \tau_{j}\left(f_{j}(u)-\lambda_{j} g_{j}(u)\right)
$$

Now apply the strict convexity of $\sum_{j=1}^{p} \tau_{j}\left(f_{j}(\bullet)-\lambda_{j} g_{j}(\bullet)\right)$ to $(28)$ to obtain

$$
(x-u)^{t} \sum_{j=1}^{p} \tau_{j} \nabla\left(f_{j}(u)-\lambda_{j} g_{j}(u)\right)<0
$$

which when added to (22) contradicts (6).

ThEOREM 4 (WEAK DUALITY). Assume that for all feasible $x$ for (FP) and all feasible $(u, \tau, y)$ for (FD2), $f_{i}(u) \geqq 0$ and $\rho_{i}$-convex and $-g_{i}(\cdot)<0$ and $\sigma_{i}$-convex, for each $i=1,2, \ldots, p$; and that $h_{j}$ is $\gamma_{j}$-convex for $j=1,2, \ldots, m$. If also either of the following is satisfied:

(a) $\quad \tau_{i}>0$ for all $i \in P=\{1,2, \ldots, p\}$ and $\sum_{i=1}^{p} \tau_{i}\left(\rho_{i}+\lambda_{i} \sigma_{i}\right)+\sum_{j=1}^{m} y_{j} \gamma_{j} \geqq 0$,

(b) $\sum \tau_{i}\left(\rho_{i}+\lambda_{i} \sigma_{i}\right)+\sum_{j=1}^{m} y_{j} \gamma_{j}>0$;

then the following cannot hold:

$$
\frac{f_{j}(x)}{g_{j}(x)} \leqq \frac{f_{j}(u)}{g_{j}(u)}=\lambda_{j} \text { for all } j \in P
$$


and

$$
\frac{f_{i}(x)}{g_{i}(x)}<\frac{f_{i}(u)}{g_{i}(u)}=\lambda_{i} \text { for some } i \in P .
$$

Remark 2. Hypothesis (a) says that if the objective function component multipliers are strictly positive, then the positive linear combination of numerators minus the non-negative linear combination of the denominators plus the non-negative linear combination of the constraints should be either convex or strongly convex. Hypothesis (b) says that the non-negative linear combination of the numerators minus the non-negative linear combination of denominators plus the non-negative linear combination of constraints should be strongly convex. The convexity conditions here are weaker than those in Theorem 3 in the sense that some functions can be weakly convex provided that the resultant linear combination is convex or strongly convex.

Proof: Suppose contrary to the result, that (29) and (30) hold. Then if $\tau_{j}>0$ for all $j \in P,(29),(30)$ and $g_{j}(\bullet)>0$ imply

$$
\sum_{j=1}^{p} \tau_{j}\left(f_{j}(x)-\lambda_{j} g_{j}(x)\right)<\sum_{j=1}^{p} \tau_{j}\left(f_{j}(u)-\lambda_{j} g_{j}(u)\right) .
$$

Also if $\tau_{j} \geqq 0$ for each $j \in P$ then again $(29),(30)$ and $g_{j}(\bullet)>0$ imply

$$
\sum_{j=1}^{p} \tau_{j}\left(f_{j}(x)-\lambda_{j} g_{j}(x)\right) \leqq \sum_{j=1}^{p} \tau_{j}\left(f_{j}(u)-\lambda_{j} g_{j}(u)\right)
$$

Now from $\tau_{j} \geqq 0, \lambda_{j} \geqq 0, f_{j}$ is $\rho_{j}$-convex and $-g_{j}$ is $\sigma_{j}$-convex for each $j \in P$ we know that $\sum_{j=1}^{p} \tau_{j}\left(f_{j}(\bullet)-\lambda_{j} g_{j}(\bullet)\right)$ is $\sum_{j=1}^{p} \tau_{j}\left(\rho_{j}+\lambda_{j} \sigma_{j}\right)$-convex. Hence (31) and (32) imply

$$
(x-u)^{t} \sum_{j=1}^{p} \tau_{j} \nabla\left(f_{j}(u)-\lambda_{j} g_{j}(u)\right)+\sum_{j=1}^{p} \tau_{j}\left(\rho_{j}+\lambda_{j} \sigma_{j}\right)\|x-u\|^{2}<0
$$

and

$$
(x-u)^{t} \sum_{j=1}^{p} \tau_{j} \nabla\left(f_{j}(u)-\lambda_{j} g_{j}(u)\right)+\sum_{j=1}^{p} \tau_{j}\left(\rho_{j}+\lambda_{j} \sigma_{j}\right)\|x-u\|^{2} \leqq 0 .
$$

Also since $x$ is feasible for (FP) and $(u, \tau, y)$ is feasible for (FD2), we have $y^{t} h(x)-$ $y^{t} h(u) \leqq 0$ and from the $\sum_{j=1}^{p} y_{j} \gamma_{j}$-convexity of $y^{t} h(\bullet)$ we obtain

$$
(x-u)^{t} \nabla y^{t} h(u)+\sum_{j=1}^{m} y_{j} \gamma_{j}\|x-u\|^{2} \leqq 0 .
$$


Adding (35) to (33) gives

$$
\begin{aligned}
& (x-u)^{t}\left(\sum_{j=1}^{p} \tau_{j} \nabla\left(f_{j}(u)-\lambda_{j} g_{j}(u)\right)+\nabla y^{t} h(u)\right)+ \\
& +\|x-u\|^{2}\left(\sum_{j=1}^{p} \tau_{j}\left(\rho_{j}+\lambda_{j} \sigma_{j}\right)+\sum_{i=1}^{m} y_{i} \gamma_{i}\right)<0 .
\end{aligned}
$$

Adding (35) to (34) yields

$$
\begin{aligned}
& (x-u)^{t}\left(\sum \tau_{j} \nabla\left(f_{j}(u)-\lambda_{j} g_{j}(u)\right)+\nabla y^{t} h(u)\right)+ \\
& +\|x-u\|^{2}\left(\sum_{j=1}^{p} \tau_{j}\left(\rho_{j}+\rho_{j} \sigma_{j}\right)+\sum_{i=1}^{m} y_{i} \gamma_{i}\right) \leqq 0 .
\end{aligned}
$$

Now applying hypothesis (a) to (36) and applying hypothesis (b) to (37) we obtain

$$
(x-u)^{t} \sum_{j=1}^{p} \tau_{j} \nabla\left(f_{j}(u)-\lambda_{j} g_{j}(u)\right)+\nabla y^{t} h(u)<0
$$

which contradicts (6).

THEOREM 5 (WEAK DUALITY). Let the conditions of Theorem 4 hold except that $y^{t} h$ be quasiconvex and hypothesis (a) and (b) become

$$
\begin{aligned}
& \text { (a') } \tau_{j}>0 \text { for all } j \in P \text { and } \sum \tau_{j}\left(\rho_{j}+\lambda_{j} \sigma_{j}\right) \geqq 0, \\
& \text { (b') } \sum_{j=1}^{p} \tau_{j}\left(\rho_{j}+\lambda_{j} \sigma_{j}\right)>0
\end{aligned}
$$

then the result of Theorem 4 holds.

Proof: The proof is similar to that of Theorem 4 except instead of using the $\sum_{j=1}^{m} y_{j} \gamma_{j}$-convexity of $y^{t} h$ we use its quasiconvexity and instead of hypotheses (a) and (b) we use hypotheses $\left(a^{\prime}\right)$ and $\left(b^{\prime}\right)$.

Corollary 2. Assume that weak duality (Theorem 3, 4 or 5) holds between (FP) and (FD2). If $\left(u^{0}, \tau^{0}, y^{0}\right)$ is a feasible solution of (FD2) such that $u^{0}$ is a feasible solution of $(F P)$, then $u^{0}$ is an efficient solution of $(F P)$ and $\left(u^{0}, \tau^{0}, y^{0}\right)$ is an efficient solution of (FD2).

Proof: The proof is similar to that of Corollary 1. 
ThEOREM 6 (STRONG DUALITY). Let $x^{0}$ be an efficient solution of (FP) and assume that $x^{0}$ satisfies a constraint qualification $[\mathbf{1}, \boldsymbol{\theta}]$ for $F P_{k}^{\prime}\left(\varepsilon^{0}\right)$ for at least one $k=1,2, \ldots, p$. Then there exist $\tau^{0} \in \mathbf{R}^{p}$ and $y^{0} \in \mathbf{R}^{m}$ such that $\left(x^{0}, \tau^{0}, y^{0}\right)$ is feasible in (FD2). If also weak duality (Theorem 3,4 or 5 ) holds between (FP) and (FD2), then $\left(x^{0}, \tau^{0}, y^{0}\right)$ is efficient for (FD2).

Proof: Since $x^{0}$ is efficient for (FP), from Lemma 2 we know that $x^{0}$ solves $\mathrm{FP}_{k}^{\prime}\left(\varepsilon^{0}\right)$ for each $k=1,2, \ldots, p$. By hypothesis $x^{0}$ satisfies a constraint qualification $[\mathbf{1}, 6]$ for $\mathrm{FP}_{k}^{\prime}\left(\varepsilon^{0}\right)$ for some $k \in P=\{1,2, \ldots, p\}$. From Kuhn-Tucker necessary conditions $[1,6]$ there exist $y \geqq 0 \in \mathbf{R}^{m}$ and $\tau_{i} \geqq 0$ for all $i \neq k$ such that

$$
\begin{aligned}
\nabla \frac{f_{k}\left(x^{0}\right)}{g_{k}\left(x^{0}\right)}+\sum_{i \neq k} \tau_{i} \nabla\left(f_{i}\left(x^{0}\right)-\epsilon_{i}^{0} g_{i}\left(x^{0}\right)\right)+y^{t} \nabla h\left(x^{0}\right) & =0 \\
y^{t} h\left(x^{0}\right) & =0 .
\end{aligned}
$$

Now we set $\lambda_{k}^{0}=\frac{f_{k}\left(x^{0}\right)}{g_{k}\left(x^{0}\right)}$ and $\lambda_{j}^{0}=\varepsilon_{j}^{0}=\frac{f_{j}\left(x^{0}\right)}{g_{j}\left(x^{0}\right)}, j \neq k$ and $\tau_{k}=\frac{1}{g_{k}\left(x^{0}\right)}$. Then $\sum_{j=1}^{p} \tau_{j}=\frac{1+g_{k}\left(x^{0}\right) \sum_{j \neq k} \tau_{j}}{g_{k}\left(x^{0}\right)}$.

Now dividing both sides of equations (38) and (39) by $\left(1+g_{k}\left(x^{0}\right) \sum_{j \neq k} \tau_{j}\right) / g_{k}\left(x^{0}\right)$ and setting

$$
\begin{aligned}
\tau_{k}^{0} & =\frac{1}{1+g_{k}\left(x^{0}\right) \sum_{j \neq k} \tau_{j}}>0, \\
\tau_{i}^{0} & =\frac{g_{k}\left(x^{0}\right) \tau_{i}}{1+g_{k}\left(x^{0}\right) \sum_{j \neq k} \tau_{j}} \geqq 0, \text { for all } i \neq k, \\
y^{0} & =\frac{g_{k}\left(x^{0}\right) y}{1+g_{k}\left(x^{0}\right) \sum_{j \neq k} \tau_{j}} \geqq 0 ;
\end{aligned}
$$

then $\left(x^{0}, \tau^{0}, y^{0}\right)$ is a feasible solution of (FD2). Efficiency of $\left(x^{0}, \tau^{0}, y^{0}\right)$ for (FD2) is now immediate from Corollary 2.

\section{REFERENCES}

[1] M.S. Bazaraa and C.M. Sluetty, Nonlinear Programming: Theory and Algorithms (John Wily, 1979).

[2] C.R. Bector, 'Duality in nonlinear fractional programming', $Z$. Oper. Res. 17 (1973), 183-193. 
[3] V. Chankong and Y.Y. Haimes, Multiobjective Decision Making: Theory and Methodology. (North -Holland, New York, 1983).

[4] R.R. Egudo, 'Efficiency and generalized convex duality for multiobjective programs', J. Math. Anal. Appl. (to appear).

[5] A.M. Geoffrion, 'Proper efficiency and the theory of vector maximization', J. Math. Anal. Appl. 22 (1968), 618-630.

[6] O.L. Mangasarian, Nonlinear Programming (McGraw-Hill, New York, 1969).

[7] B. Mond and T. Weir, 'Generalized concavity and duality', in Generalised Concavity in Optimization and Economics, ed. S. Schaible and W.T. Ziemba, pp. 263-279 (Academic Press, 1981).

[8] B. Mond and T. Weir, 'Duality for fractional programming with generalized convexity conditions', J. Inform. Optim. Sci. 3 (1982), 105-124.

[0] S. Schaible, 'Fractional programming. 1. Duality', Management Sci. 22 (1976), 858-867.

[10] S. Schaible, 'Duality in fractional prograinming: a unified approach', Oper. Res. 24 (1976), 452-461.

[11] C. Singh, 'A class of multiple-criteria fractional programming problems', J. Math. Anal. Appl. 115 (1968), 202-213.

[12] J.P. Vial, 'Stroug convexity of sets and functions', J. Math. Econom. 0 (1982), 187-205.

[13] J.P. Vial, 'Strong and weak convexity of sets and functions', Math. Oper. Res. 8 (1983), 231-259.

[14] T. Weir, 'A duality theorem for a multiobjective fractional optimization problem', Bull. Austral. Math. Soc. 34 (1986), 415-425.

[15] T. Weir, 'A dual for a multiple objective fractional programming problem', J. Inform. Optim. Sci. 7 (1986), 261-269.

Scliool of Applied Science

Gippsland lnstitute of Advanced Education

Switchback Rd

Churchill Vic. 3852

A ustralia 